\title{
Effects of attribute identitication training on rule effects in an attribute identification transfer task*
}

\author{
LINDA J. INGISON $\dagger$ \\ University of Wisconsin, Madison, Wisconsin 53706
}

\begin{abstract}
The hypothesis that attribute identification (AI) pretraining would reduce or eliminate rule effects in AI tasks was investigated. Ss were given 0,4 , or $8 \mathrm{AI}$ problems based on the same rule prior to transfer to a final AI problem. Results indicated that familiarity with AI problems was not a key factor in eliminating rule effects in transfer. Implications for a two-component model of concept learning were discussed.
\end{abstract}

A current model of concept learning (cf. Haygood \& Bourne, 1965) postulates that the rule learning (RL) and attribute identification (AI) components of concept problems involve independent processes. Research generated within this model, however, has not been entirely supportive. Specifically, rule effects have repeatedly been demonstrated in AI tasks (Haygood \& Bourne, 1965; Bourne \& Guy, 1968b; Di Vesta \& Walls, 1969) despite the fact that, logically, rule effects should be expected only in RL tasks.

Assuming the validity of a model of independent processes, the presence of rule effects in AI tasks may be due to methodological or procedural problems. Two likely sources of difficulty immediately present themselves. First, typical AI instructions may not succeed in conveying the rule to $S$, especially when highly unfamiliar rules (e.g., biconditional) are used. Evidence that rule familiarity alone is not responsible for producing AI rule effects has recently been found, however (Ingison, 1974).

A second explanation of AI rule effects may be given in terms of S's strategy or approach to concept problems. It is possible that strategies specific to the underlying rule must be developed with practice on successive AI tasks in order to reduce or eliminate rule effects. Laughlin and his co-workers (1968; Laughlin \& Jordan, 1967) have identified optimal AI strategies in the selection paradigm. These findings suggest that optimal reception strategies may exist. If so, strategies appropriate to familiar rules (e.g., conjunctive) may be more highly developed than those strategies appropriate

*This research was funded by the Wisconsin Research and Development Center for Cognitive Learning, supported in part as a research and development center by funds from the National Institute of Education. The opinions herein do not necessarily reflect the position or policy of the National Institute of Education, and no official endorsement by the National Institu te should be inferred. Center No. NE-C-00-3-0065. This paper was sponsored by Peter D. Kepros, who takes full editorial responsibility for its contents.

tR equests for prints may be sent to Linda J. Ingison Wisconsin Research and Development Center, 1025 West Johnson Street, University of Wisconsin, Madison, Wisconsin 53706. to difficult rules. Thus, naive Ss given AI problems based on difficult or unfamiliar rules may be required not only to identify the relevant attributes, but also to learn the appropriate rule strategy. It is possible that varying levels of familiarity with particular rules in AI may contribute to obtained rule effects. The present experiment was designed to test the hypothesis that differences in $\mathrm{AI}$ familiarity account for AI rule effects.

\section{METHOD}

\section{Subjects and Design}

Ss were 3612 th-grade volunteers from the public schools of a semirural community in Wisconsin. Each $\mathrm{S}$ was assigned to one of six independent conditions by means of six randomized sequences designed to place one $\mathrm{S}$ in each cell before placing a second $\mathrm{S}$ in any cell. The experimental design was a 2 (rules-Dj or Cd) by 3 (pretraining levels $-0,4$, or 8 pretraining problems prior to a transfer AI task) factorial. A total of six Ss were assigned to each cell described by the design.

\section{Stimuli and Problems}

The stimulus population, the selection of relevant attributes, and the arrangement of stimulus sequences were described earlier (Ingison, 1974). A total of nine AI problems (up to eight practice plus one test problem) were required by the design.

\section{Procedure}

Each $\mathrm{S}$ was tested individually. Instructions were read to $\mathrm{S}$, and appropriate example cards were provided by E. Stimuli were presented on slides and projected by means of a Kodak Carousel projector. A modified reception paradigm, described earlier (Ingison, 1974) was employed. The Ss responded verbally on test trials, and E provided feedback for S. Each slide remained on the screen for approximately $4-5 \mathrm{sec}$. The Ss were given up to 72 test trials on each problem to reach a criterion of 16 correct test trials in a row. Those Ss not reaching criterion at this time were given the solution by $\mathrm{E}$ and required to demonstrate this solution by reaching the criterion of 16 correct. At this time, $S$ was taken to the next problem. Thus, no Ss were eliminated from the analysis.

\section{RESULTS}

Transfer performance was assessed by a 3 (pretraining levels $-0,4$, and $8 \mathrm{AI}$ practice problems) by 2 (rules-Dj or $\mathrm{Cd}$ ) analysis of variance on errors to criterion. Table 1 
Table 1

Mean Errors for the Nonsignificant Interaction of Pretraining by Rule

\begin{tabular}{ccrr}
\hline $\begin{array}{c}\text { Pretraining } \\
\text { Problems }\end{array}$ & Rule Dj & Rule Cd & \\
\hline 0 & 9.33 & 18.33 & 13.83 \\
4 & 3.33 & 8.17 & 5.75 \\
8 & 4.33 & 4.83 & 4.58 \\
& 5.67 & 10.44 & \\
\hline
\end{tabular}

presents the mean errors for each condition. Contrary to the prediction that increasing amounts of AI training would significantly reduce or eliminate rule effects in $\mathrm{AI}$, the interaction of pretraining and rule did not reach significance, $F(2,30)=1.57, p<.22$, indicating that rule effects shown by Ss at all pretraining levels were similar.

The main effect of pretraining reached significance, $\mathrm{F}(2,30)=8.84, \mathrm{p}<.001$. Tukey HSD tests were computed, revealing significant differences only between the 0-pretraining problem condition and all others, $p<.01$. These data indicate that as few as four AI training problems is sufficient to significantly reduce errors on subsequent tasks.

The main effect of rule also attained significance, $\mathrm{F}(1,30)=5.97, \mathrm{p}<.02$. Error means for this effect, shown in Table 1, reveal the relative difficulty of the $\mathrm{Cd}$ rule. No other effects reached significance. In sum, the results indicate that, at least for the pretraining levels employed in the present study, rule effects are not eliminated through AI training.

\section{DISCUSSION}

The major prediction in the present study was that pretraining on several AI tasks involving the same underlying rule would reduce or eliminate rule effects in a subsequent AI task. As evidenced by the absence of a Rule by Pretraining interaction, the results show no clear effect for various levels of AI training in reduction of rule differences. Ty pical rule effects were present in transfer for all pretraining conditions. Previous work (Ingison, 1974) has shown that rule differences in AI are not due to familiarity with the rule perse. The present results further indicate that rule effects are not eliminated by practice on AI problems (at least for the pretraining levels utilized in the present experiment).

Taken together, these results call into question the validity of a two-component model of concept learning. If rule effects in AI tasks are apparent despite training on $\mathrm{RL}$ or AI problems, it is difficult to identify manipulations which would eliminate them. The remaining possibilities are overtraining on $\mathrm{RL}$ or $\mathrm{AI}$ problems or training on some repertoire of skills such as truth table coding, RL (or AI) training on a variety of rules, etc.

Should overtraining or a varied repertoire of skills fail to reduce rule effects in $\mathrm{AI}$, models proposing independent processes for RL and AI may have to be revised to account for a third component: the interaction of $\mathrm{RL}$ and $\mathrm{AI}$. Further, past and future research based on the two-component model will have to be interpreted cautiously (at least for certain variables) in order to avoid the erroneous assumption that obtained results are attributable solely to independent R L or AI components and processes.

\section{REFERENCES}

Bourne, L. E., Jr., \& Guy, D. E. Learning conceptual rules: II. The role of positive and negative instances. Journal of Experimental Psychology. 1968b. 7 7, 488-494.

Di Vesta, F. J., \& Walls, R. T. Rule and attribute identification in children's attainment of conjunctive and disjunctive concepts. Journal of Experimental Psychology, 1969, 80, 498-504.

Haygood, R. C., \& Bourne, L. E., Jr. Attribute- and rule-learning aspects of conceptual behavior. Psychological Review, 1965 72, 175-195.

Ingison, L. J. Effects of rule pretraining on rule effects in an attribute identification task. Bulletin of the Psychonomic So ciety, 1974, 3, 355-357.

Laughlin, P. R. Focusing strategy for eight concept rules. Journal of Experimental Psychology, 1968, 77, 661-669.

Laughlin, $P$. R \& Jordan, $R$. $M$. Selection strategies in conjunctive, disjunctive, and biconditional concept attainment. Journal of Experimental Psychology, 1967, 75, 188-193.

(R eceived for publication May 17, 1974.) 